\title{
Foliar microbiome transplants confer disease resistance in a critically-endangered plant
}

\author{
Geoffrey Zahn ${ }^{1}$, Anthony S Amend ${ }^{\text {Corresp. } 2}$ \\ 1 Biology Department, Utah Valley University, Orem, Utah, United States \\ 2 Botany Department, University of Hawaii at Manoa, Honolulu, Hawaii, United States \\ Corresponding Author: Anthony S Amend \\ Email address: amend@hawaii.edu
}

There has been very little effort to incorporate foliar microbiomes into plant conservation efforts even though foliar endophytes are critically important to the fitness and function of hosts. Many critically endangered plants that have been extirpated from the wild are dependent on regular fungicidal applications in greenhouses that cannot be maintained for remote out-planted populations, which quickly perish. These fungicides negatively impact potentially beneficial fungal symbionts, which may reduce plant defenses to pathogens once fungicide treatments are stopped. Using the host/parasite system of Phyllostegia kaalaensis and Neoerysiphe galeopsidis, we conducted experiments to test total foliar microbiome transplants from healthy wild relatives onto fungicide-dependent endangered plants in an attempt to mitigate disease and reduce dependency on fungicides. Plants were treated with total microbiome transplants or cultured subsets of this community and monitored for disease severity. High-throughput DNA screening of fungal ITS1 rDNA was used to track the leaf-associated fungal communities and evaluate the effectiveness of transplantation methods. Individuals receiving traditionally isolated fungal treatments showed no improvement, but those receiving applications of a simple leaf slurry containing an uncultured fungal community showed significant disease reduction, to which we partially attribute an increase in the mycoparasitic Pseudozyma aphidis. These results were replicated in two independent experimental rounds. Treated plants have since been moved to a native habitat and, as of this writing, remain disease-free. Our results demonstrate the effectiveness of a simple low-tech method for transferring beneficial microbes from healthy wild plants to greenhouse-raised plants with reduced symbiotic microbiota. This technique was effective at reducing disease, and in conferring increased survival to an out-planted population of critically endangered plants. It was not effective in a closely related plant. Plant conservation efforts should strive to include foliar microbes as part of comprehensive management plans. 
1 Foliar microbiome transplants confer disease resistance in a critically-endangered plant 2

3 Geoffrey Zahn ${ }^{1} \&$ Anthony Amend ${ }^{2 *}$

$4 \quad{ }^{1}$ Utah Valley University, Biology Department, 800 W. University Parkway, Orem, UT 84058

5 '2University of Hawai'i at Manoa, Botany Department, 3190 Maile Way, Room 101, Honolulu, HI 96822, USA

6 * Corresponding Author: amend@hawaii.edu, +1-808-956-8369

7

8 Tables and Figures:

9 Main figures - 3 (Grayscale); Tables - 0; Supporting figures - 2 (Color); Supporting data - 1 (.zip archive) 
10 Foliar microbiome transplants confer disease resistance in a critically-endangered plant

11 Abstract

There has been very little effort to incorporate foliar microbiomes into plant conservation efforts even though foliar endophytes are critically important to the fitness and function of hosts. Many critically endangered plants that have been extirpated from the wild are dependent on regular fungicidal applications in greenhouses that cannot be maintained for remote out-planted populations, which quickly perish. These fungicides negatively impact potentially beneficial fungal symbionts, which may reduce plant defenses to pathogens once fungicide treatments are stopped. Using the host/parasite system of Phyllostegia kaalaensis and Neoerysiphe galeopsidis, we conducted experiments to test total foliar microbiome transplants from healthy wild relatives onto fungicide-dependent endangered plants in an attempt to mitigate disease and reduce dependency on fungicides. Plants were treated with total microbiome transplants or cultured subsets of this community and monitored for disease severity. High-throughput DNA screening of fungal ITS1 rDNA was used to track the leaf-associated fungal communities and evaluate the effectiveness of transplantation methods. Individuals receiving traditionally isolated fungal treatments showed no improvement, but those receiving applications of a simple leaf slurry containing an uncultured fungal community showed significant disease reduction, to which we partially attribute an increase in the mycoparasitic Pseudozyma aphidis. These results were replicated in two independent experimental rounds. Treated plants have since been moved to a native habitat and, as of this writing, remain disease-free. Our results demonstrate the effectiveness of a simple lowtech method for transferring beneficial microbes from healthy wild plants to greenhouse-raised plants with reduced symbiotic microbiota. This technique was effective at reducing disease, and in conferring increased survival to an out-planted population of critically endangered plants. It was not effective in a closely related

31 plant. Plant conservation efforts should strive to include foliar microbes as part of comprehensive management 32 plans.

\section{Introduction}

So far, foliar fungal endophytes have been found in every natural plant examined (Petrini 1986; 
37 negatively, as leaf-associated fungi that do not show pathogenicity (Hardoim et al. 2015). This definition is contextually dependent on a wide range of factors that influence how leaf-inhabiting fungi interact with their plant hosts, including fungal genotype (Rudgers, Fischer \& Clay 2010), and climatic change such as increased drought frequency (Desprez-Loustau et al. 2006).

The fungi that inhabit the phyllosphere are likely as important to plant health as are belowground fungi (Vicari, Hatcher \& Ayres 2002; Herre et al. 2007; Porras-Alfaro \& Bayman 2011). Evidence suggests that naturally occurring fungal foliar endophytes partially determine disease severity in agricultural systems (Xia et al. 2015; Ridout \& Newcombe 2016), tropical trees (Arnold et al. 2003), and Populus models (Busby, pathogenic species, competition for resources, and/or by altering plant host defenses. Therefore, endophytes may be most usefully thought of as modifiers of plant disease (Busby, Ridout \& Newcombe 2015), and/or insect herbivory (Breen 1994; Hartley \& Gange 2009) rather than as simply transitively "non-pathogenic."

This perspective has led to many biocontrol efforts that (with varied success) seek to reduce disease severity by using beneficial foliar endophytes, particularly in commercially important plants (Viterbo et al. 2002; Kiss 2003; Miller et al. 2004; Bressan \& Borges 2004; Gafni et al. 2015; Borges, Saraiva \& Maffia 2015). However, to date, there seems to be less effort to apply this knowledge to plant conservation efforts.

The work that has addressed any microbial components of plant conservation has focused mostly on belowground plant-microbe relationships, especially on arbuscular mycorrhizal symbioses (e.g., Requena et al. 2001; Gemma, Koske \& Habte 2002; Zubek et al. 2008; Harris 2009; Ferrazzano \& Williamson 2013; Rigg et al. 2016). These studies, and others, have shown that soil microbes can play a large role in plant success in a

57 given habitat, but relatively less attention has been granted to the aboveground microbes that interact with 58 plants. liberation and uptake) (Heilmann-Clausen et al. 2015), but these services remain unexplored with regard to foliar fungi. Here, we examine how manipulating foliar endophytes modifies plant disease on critically be used in conservation. 

of O'ahu in Hawai'i. The genus Phyllostegia, found only in the Hawai'ian Islands, represents a radiation from presumably one introduction of an allopolyploid ancestor and are phylogenetically nested within the North American genus Stachys (Baldwin \& Wagner, 2010). This ancestor has since radiated into 32 recognized species of Phyllostegia. Of these, 14 are listed as critically endangered (The IUCN Red List of Threatened Species. Version 2017-2) and most of the others are presumed to be extinct. Currently extirpated in the wild, $P$. kaalaensis only exists as populations in two greenhouse facilities, one managed by the state of Hawai'i and one by the U.S. Army. Although clonal propagation is readily achieved out-planting efforts have yielded no longterm success, defined by survival of at least one year and active recruitment of new plants (Weisenberger \& Keir 2012). In the greenhouse environment, $P$. kaalaensis is highly susceptible to infection by the powdery mildew Neoerysiphe galeopsidis, which can lead to total mortality within 30 days if untreated (Matthew Keir, personal communication). This leaves greenhouse-raised plants dependent on regular applications of topical fungicide (Mancozeb, DuPont, Wilmington, DE, USA). is impractical for out-planted populations in remote sites. Additionally, fungicide applications can have undesirable effects on beneficial fungal endophytes (Karlsson et al. 2014). Thus it is likely that the fungicide used on greenhouse-raised $P$. kaalaensis individuals are inhibiting pathogen antagonists as well as the pathogen. This might lead to plants being reintroduced to their native range with reduced colonization of potentially beneficial foliar fungi, possibly making them more susceptible to environmental pathogens or otherwise maladapted to natural environments. We hypothesized that re-establishing endophyte communities within foliar tissues would increase disease resistance and improve out-planting success. survival in the wild.

\section{Methods}




\section{Experimental design and overview}

92 The experiment tested the disease modification properties of fungal endophyte isolates and uncultured

93 fungi from a slurry of surface-sterilized leaves obtained from wild healthy relative, Phyllostegia hirsuta. P.

94 hirsuta is another endangered mint, whose range overlaps $P$. kaalaensis, and it was chosen as a microbial

95 donor since outplanting efforts have yielded recent success in re-establishing stable wild populations (New

96 plant recruitment for at least one year, Matthew Keir, personal communication). We chose two endangered

97 plant species, $P$. kaalaensis and $P$. mollis, as microbial recipients due to their critically endangered status and

98 the fact that extant populations require weekly fungicide applications. The logistics of working with critically

99 endangered plants limited the scope of the experiment. Only $\sim 18$ individuals per species were available at a

100 time, so we selected three treatments: a slurry of leaves from wild Phyllostegia hirsuta containing uncultivated

101 fungi, a slurry of spores from eleven cultured endophyte isolates representing a readily-cultivable subset of the

102 leaf slurry fungi, and a sterile water control.

103 We exposed all plants to the $N$. galeopsidis pathogen, and disease severity was observed until plant

104 mortality. Throughout the experiment, DNA was extracted from surface-sterilized leaves to track endophytic

105 fungal community composition. We repeated the entire experiment a second time with a new set of 18 plants in

106 order to confirm the initial findings and to assess reproducibility. At the conclusion of both experimental

107 rounds, we performed a final control round consisting of two treatments, a leaf slurry and a leaf slurry filtered

108 through 0.2 um to remove fungi and bacteria, to confirm that observed effects were attributable to biota and

109 not to phytochemicals present in the leaf slurry. In the subsections below, we present methods that first outline

110 plant and inoculum preparation, describe the experimental trials, and explain the workflow for wet lab work

111 and bioinformatic analyses.

112

113 Plant acquisition

114 We acquired P. kaalaensis and P. mollis individuals from the Oahu Army Natural Resources Program

115 (OANRP) under authorization of the USFWS on the US Army's permit (TE-043638-10). Experimental plants

116 were grown from cuttings of greenhouse individuals from 4 clonal lines and were randomly assigned to

117 experimental groups. Plants arrived in 4-inch pots of soil-less medium (Sunshine \#4, SunGro Horticulture) and 
118 remained in these pots for the duration of the experiment. Though greenhouse populations are dependent on

119 regular chemical treatments, these individuals had not been treated with fungicide or insecticide since cuttings

120 were taken ( $\sim 8$ weeks). Plants were watered from below with sterile D.I. water every other day for the duration

121 of the trials, and humidity was passively controlled by keeping a shallow pan of sterile water open on the floor

122 of the growth chambers.

123

124 Inoculum and pathogen acquisition and preparation

125 Fungal isolates were obtained by placing small cuttings of surface-sterilized $P$. hirsuta leaves,

126 collected from the wild, on MEA medium amended with Streptomycin and Kanamycin (Supporting

127 Information). After three weeks of growth, we identified 11 morphologically dissimilar sporulating isolates by

128 Sanger sequencing of the ITS1-28S region of ribosomal-encoding DNA amplified with ITS1F (5'-

129 CTTGGTCATTTAGAgGAAGTAA-3') (Gardes \& Bruns, 1993) and TW-13 (5’-

130 GGTCCGTGTTTCAAGACG-3') (White et al., 1990). Molecular identification supported the separation of

131 the morphologically-distinct isolates. These isolate cultures were flooded with sterile water, gently shaken to

132 release spores, and spores were pooled in equal concentrations $\left(2.3 * 10^{6}\right.$ cells $\left./ \mathrm{mL}\right)$ to compose the "isolate

133 slurry."

134 The leaf slurries were obtained by blending surface-sterilized $P$. hirsuta leaves in sterile water for 1

135 minute in a Waring Laboratory Blender and then filtering through a $100 \mu \mathrm{m}$ membrane to remove large

136 particles. The resulting "leaf slurry" contained the natural endophytic community of $P$. hirsuta and was used

137 without further processing.

138

139 Incubation and pathogen challenge

140 Plants were kept in Percival growth chambers at 21 degrees C under 12 hours of light per day (550

$141 \mu$ moles PAR $\mathrm{m}^{2} \mathrm{~s}^{-1}$ ) and watered twice weekly. We used a foliar spray method similar to Posada et al. (Posada

142 et al. 2007) to inoculate leaves. Briefly, inoculation was performed with a hand sprayer, applying

143 approximately $5 \mathrm{ml}$ of inoculum per plant, per application period, and plants were covered by plastic bags for 
14424 hours immediately after to increase humidity. To improve the efficacy of any potential biocontrol agents

145 (Filonow et al. 1996), plants were inoculated weekly for three weeks prior to pathogen exposure. After three

146 weeks, the pathogen was introduced by placing an infected $P$. kaalaensis leaf from the OANRP greenhouse in

147 the air intake of the growth chambers. Weekly, all the leaves of each plant were visually inspected for signs of

148 infection and the total proportion of infected leaf area was recorded as a measure of disease severity.

DNA methods

We extracted DNA from the inoculum sources and from surface sterilized leaf punches when the plants arrived, in the middle (immediately after the first visible signs of powdery mildew infection), and at the end of incubations. Two leaf punches from each plant were made with a $1 \mathrm{~cm}$ diameter sterile hole punch, avoiding visibly infected areas, and were surface-sterilized by shaking in $1 \%$ bleach for 1 minute, $70 \%$ ethanol for 2 minutes, and two rinses in sterile water for 2 minutes each. Inoculum slurries were centrifuged for 10 minutes at 10,000 RCF and resultant pellets were retained for DNA extraction. DNA was extracted from surface-sterilized leaf punches and inoculum pellets with MoBio Powersoil kits (QIAGEN, Venlo, The 158 Netherlands). to always obtain two leaf disks from each plant. Therefore, for each sampling period, we pooled leaf disks within each group and randomly selected two plugs for each of three extractions.

163 Illumina adaptors (Caporaso et al. 2011) using the following protocol: 98 2min; 22 cycles of: 98 15s, 52 30s,

$1647230 \mathrm{~s} ; 72$ 2min). After 22 cycles, the PCR product was diluted $1: 12$ and $1 \mu \mathrm{L}$ of this was used as a template

165 for 8 more rounds of PCR with a 60 deg annealing temperature in which bi-directional barcodes bound to

166 reverse complimented Illumina adaptors acted as primers. Resulting barcoded libraries were cleaned,

167 normalized, and sequenced with the Illumina MiSeq platform (V3 chemistry, 2 x $300 \mathrm{bp}$ ).

168

169 Bioinformatics/Statistics 
171 chimera removal, followed by extraction of the ITS1 region and open-reference OTU picking. Illumina reads

172 were demultiplexed by unique barcode pairs and forward and reverse reads were merged with Pear (Zhang et

173 al. 2014). Reads that were successfully assembled were then quality screened with the fastx_toolkit

174 (http://hannonlab.cshl.edu/fastx_toolkit/index.html) to remove reads shorter than 200bp or longer than $500 \mathrm{bp}$

175 and those that contained any bases with a quality score lower than 25.

176 Quality-screened reads were then checked for chimeras both de novo and against the UNITE-based

177 chimera database (Nilsson et al. 2015; downloaded 31.01.2016) to remove any putative chimeric sequences

178 with VSearch 1.9.1 (Rognes et al. 2016). Non-chimeric sequences (those passing both screening steps) were

179 subsequently run through ITSx (Bengtsson-Palme et al. 2013) to extract fungal ITS1 sequences (i.e., only the

180 ITS1 region of sequences determined to be fungal in origin).

181 OTUs were clustered at 97\% similarity from screened ITS1 sequences with the uclust algorithm

182 (Edgar 2010) wrapped within the open-reference OTU picking workflow of QIIME version 1.9.1 (Caporaso et

183 al. 2010) and taxonomy was assigned against the dynamic UNITE fungal database (Kõljalg et al. 2013)

184 version 1.31.2016. The resultant OTU table was then filtered in R (version 3.3.3) to remove singletons and

185 OTUs that occurred in a given sample at less than $0.1 \%$ of the abundance of the maximum read abundance to

186 control for index bleed-over. Finally, reads present in extraction and PCR negatives were subtracted from

187 samples and the OTU table was subsampled to a depth of 8000 reads per sample with the vegan package in R

188 (Okansen et al. 2016) to determine normalized relative abundance. Bray-Curtis community dissimilarity

189 measures were performed on rarefied data with the vegdist function of the vegan package in R.

190 We initially identified potentially beneficial OTUs (i.e., those associated with reduced disease

191 severity) with the indicspecies R package (Cáceres \& Legendre 2009) on samples grouped by quartile values

192 into bins of disease coverage, measured as percent of leaf surface area infected. OTUs that were significantly

193 correlated with low-disease samples were then tested as predictors of N. galeopsidis relative abundance and

194 disease severity in a generalized linear model with a binomial family and logistic link function.

195

196 Results 
197

198

199

200

201

202

203

204

205

206

207

208

209

210

211

212

213

214

215

216

217

218

219

220

221

222

\section{Disease progression and treatment effectiveness}

The fungal isolate slurry treatment did not reduce disease severity in either plant species during either experimental round, whereas the wild leaf slurry reduced disease severity in P. kaalaensis in both trials. (Binomial GLM; Round 1: $\mathrm{P}=0.0029$, Pseudo-R2 = 0.808; Round 2: $\mathrm{P}=0.0015$, Pseudo-R2 $=0.745$ ). The two experimental rounds showed congruent results, though on different time scales. Plants in the first round rapidly succumbed to $N$. galeopsidis infection after about 30 days, but during the second round, disease took longer to manifest with infections showing up at $\sim 30$ days, and plant mortality by $\sim 90$ days. P. mollis individuals did not respond to either treatment (Fig. 1) and are excluded from further analyses. The additional control round (performed only with P. kaalaensis) demonstrated that removing biota from the wild leaf slurry with a $0.2 \mu \mathrm{m}$ filter eliminated the beneficial effects, with the unfiltered slurry showing significantly less disease severity than the filtered slurry (Binomial GLM; $\mathrm{P}=0.0034$ ).

\section{Bioinformatics}

The sequencing run returned 2,273,484 raw forward and reverse reads for analyses. Of these, 2,136,144 were successfully merged. After quality filtering, ITS extraction, and chimera removal, 1,629,699 reads remained, yielding 199 OTUs after singleton removal. Eight OTUs accounted for $\sim 94 \%$ of all reads, and a single OTU (N. galeopsidis) accounted for $\sim 76 \%$ of all reads.

\section{Fungal communities in slurries and leaves}

The vast majority of sequences from the wild leaf slurries were identified as the pathogen, $N$. galeopsidis. This was surprising, given that the $P$. hirsuta individuals donating to this slurry showed no signs of powdery mildew infection, and considering that the wild leaf slurry was the treatment shown to reduce $N$. galeopsidis disease severity. Twenty-one other OTUs were detected in the leaf slurry inoculum over both rounds, but none of these, other than Neopestalotiopsis saprophytica, comprised greater than $5 \%$ relative abundance (See Fig. 2). Sequence libraries of fungal isolate slurry samples contained 8 OTUs (representing 8 of the 11 isolates added to the slurry) and were similarly dominated by a single taxon, Alternaria alternata. 
223 Although three taxa were not recovered by sequencing, all 11 fungal taxa were successfully re-isolated from

224 the slurry on MEA media.

$225 \quad$ N. galeopsidis OTU relative abundance correlated strongly with increased disease severity in plants

226 (Binomial GLM; $\mathrm{P}=0.0020$, Pseduo- $\mathrm{R} 2=0.75$ ). Both disease severity and $N$. galeopsidis relative abundance

227 were negatively correlated with the relative abundance of a single taxon, the mycoparasitic basidiomycete

228 yeast Pseudozyma aphidis (Binomial GLM; Disease Severity: $\mathrm{P}=0.0112 ;$; N. galeopsidis rel. abundance: $\mathrm{P}=$

229 0.0071). P. aphidis was found in low relative abundance in plant leaves from all treatment groups prior to

230 experimental inoculations, but just after the first pathogen infections were visible it was significantly more

231 abundant in plants receiving the wild leaf slurry. Individuals with greater relative abundance of $P$. aphidis

232 showed sharply reduced infection severity (Fig. 3).

233 Eleven OTUs (other than $N$. galeopsidis) transferred from the leaf slurry onto plant leaves were still

234 detected halfway through the growth periods, while only six were detected at the end of the study. Pathogen

235 infection load similarity was a strong driver of community similarity (ANOVA: $P<0.00005$, $R 2=0.481$ ). Plants

236 with very high and very low infection severities hosted fungal communities that were more similar than plants

237 with intermediate infection severities. Though this was temporally confounded (infection severity and time are

238 not independent) the trend toward community convergence was driven largely by $N$. galeopsidis proliferation

239 and infection (SI Figs 1, 2).

240

241 Outplanting

242 Six healthy P. kaalaensis individuals from the leaf slurry treatment showing no sign of pathogens

243 were out-planted in April 2016 in a native habitat for monitoring. As of August 2017 they have remained

244 disease-free, and are now the only extant population of $P$. kaalaensis in the wild. The out-planting site is less

245 than $1 \mathrm{~km}$ from the location of the $P$. hirsuta from which we obtained the leaf slurry inoculum, so it is

246 presumed that there are ample $N$. galeopsidis propagules locally. This reinforces the conclusion that the

247 microbiome transplantations are serving to protect out-planted individuals from the pathogen.

248

249 


\section{Discussion}

251

This study demonstrates that foliar endophytes modify plant disease, and can be used in endangered

252 plant conservation, much as they have been for agriculturally important plants. The low-tech method of

253 spraying plants with a slurry of leaves from healthy wild relatives (containing many uncultured/unculturable

254 fungal taxa) outperformed inoculations of fungal isolates, suggesting that biodiversity was important for the

255 functional relevance of the inoculated microbes.

256 The leaf slurry treatment reduced disease severity in plants despite that the very pathogen we were

257 trying to mitigate dominated the sequencing library. The donor plants showed no obvious signs of $N$.

258 galeopsidis infection, and it is possible that the strain present in the slurry differed from the strain causing $P$.

259 kaalaensis mortality. However, we were unable to determine this from our data since all ITS1 reads assigned

260 to $N$. galeopsidis were nearly identical to the voucher sequence for the pathogen found on Oahu (with the

261 exception of four singleton variants that differed slightly but were removed because each only had one read;

262 see Supporting Information). Further, the ITS1 reads assigned to N. galeopsidis from both the slurry and

263 infected plants were identical. N. galeopsidis is known to cause disease in all studied species of Phyllostegia,

264 in the ancestral genus Stachys within North America, (Glawe, 2007) and a closely related strain of $N$.

265 galeopsidis has been reported on Stachys hosts from Eastern Asia (Heluta et al., 2010). It is not known how recently N. galeopsidis arrived in Hawai'i or whether it came with the original ancestor to modern Phyllostegia 267 species.

The relative abundance of the mycoparasitic fungus, $P$. aphidis, is a plausible explanation for the decrease in disease severity since $P$. aphidis has previously been shown to be antagonistic against powdery mildews (Buxdorf, Rahat \& Levy 2013; Gafni et al. 2015), and to reduce the incidence of plant disease (Barda et al. 2015). Its genome contains genes for chitinase, two chitinase-related genes, and other cell-wall degrading

272 proteins (Lorenz et al. 2014). Additionally, it has been shown to promote plant health, possibly via heavy

273 siderophore production which potentially limits pathogen growth by chelating available iron (Fu et al. 2016). 
276 meaning that it was either not present or that it was present at undetectably low relative abundance given the

277 numerical dominance of $N$. galeopsidis reads. In this case, it seems likely that a diverse assemblage of fungi

278 (and/or bacteria) was responsible for the relative increase in P. aphidis relative abundance in plants sprayed

279 with the leaf slurry. At the end of the study, light microscopy of necrotic lesions taken from plants treated with

280 the leaf slurry appeared to reveal $P$. aphidis attacking $N$. galeopsidis spores, though this is not conclusive (See

281 supporting information). The ability to determine the success of comprehensive fungal microbiome

282 transplantations was limited by the dominance of the pathogen in final amplicon sequences. Taxa with low

283 relative abundances were less likely to be detected as $N$. galeopsidis reads proliferated at the end of the growth

284 periods, but the 11 taxa that were detected halfway through the trial and 6 that were detected at the end indicate

285 that the simple indiscriminate transplantation of leaf microbiota was successful in establishing a diverse

286 assemblage of uncultured endophytes.

287 Primer biases or preferential Illumina clustering for shorter sequences were potentially responsible for

288 the dominance of A. alternata in isolate inoculum reads, despite spore-count normalization. Adams, et al.

289 (2013) demonstrated that abundant fungal ITS reads have the potential to swamp out known community

290 members. We did not observe the same ameliorative effect of $P$. aphidis in the other endangered plant species,

291 P. mollis, which implies that plant-microbe interactions were important for our outcome. Barda et al. (2015)

292 showed that $P$. aphidis was capable of inducing pathogenesis-related genes and triggering an induced pathogen

293 resistance response in tomato plants and it is possible that induced host plant defenses instead of, or in addition

294 to, direct antagonism played a part in the positive outcome for P. kaalaensis. Further, it is possible that other

295 microbial taxa, such as bacteria, were instrumental in producing the ameliorative effect of the leaf slurry.

296 This study reinforces the idea plants are not just plants; they are a complex assemblage of organisms

297 (Porras-Alfaro \& Bayman 2011), and should be considered as such when planning conservation approaches.

298 Since they are integral components of plant health, foliar fungi should be a key aspect of management plans for

299 endangered plants, particularly those suffering from pathogen-induced mortality. This simple approach of

300 wholesale transplantation of a microbiome conferred disease resistance to a plant on the brink of extinction,

301 and may be usefully applied to other plants.

302 


\section{Data Accessibility}

304 Raw Illumina sequences of ITS amplicons used in this study have been deposited in the Sequence

305 Read Archive; BioProject Accession: PRJNA342669. Sanger sequences of fungal isolates have been deposited

306 in Genbank under the Accessions: KX988291 - KX988301. Plant disease data, additional figures, sequence

307 alignments, analysis code, and all raw data are available in the supplemental materials.

308

309 Acknowledgements

310 We would like to gratefully acknowledge the US Army for logistical support: K Kawelo, M Kier, L

311 Weisenberger and V Costello from U.S. Army Garrison - Hawai'i’s O'ahu Army Natural Resources Program

312 (OANRP) for invaluable field and greenhouse assistance, for their expertise in rare plant conservation, and for

313 providing the plants used in this study, along with B Sedlmayer for assisting with disease monitoring.

314

315 


\section{References}

317 Adams, R. I., Amend, A. S., Taylor, J. W., \& Bruns, T. D. (2013). A Unique Signal Distorts the

318

319

320

321

322

323

324

325

326

327

328

329

330

331

332

333

334

335

336

337

338

339

340

341

342

343

344 345

Perception of Species Richness and Composition in High-Throughput Sequencing Surveys of Microbial Communities: a Case Study of Fungi in Indoor Dust. Microbial Ecology, 66, 735-741.

Arnold, A.E., Mejía, L.C., Kyllo, D., Rojas, E.I., Maynard, Z., Robbins, N. \& Herre, E.A. (2003) Fungal endophytes limit pathogen damage in a tropical tree. Proceedings of the National Academy of Sciences, 100, 15649-15654.

Barda, O., Shalev, O., Alster, S., Buxdorf, K., Gafni, A. \& Levy, M. (2015) Pseudozyma aphidis Induces Salicylic-Acid-Independent Resistance to Clavibacter michiganensis in Tomato Plants. Plant Disease, 99, 621-626.

Baldwin BG., Wagner WL. 2010. Hawaiian angiosperm radiations of North American origin. Annals of Botany 105:849-879. DOI: 10.1093/aob/mcq052.

Bengtsson-Palme, J., Ryberg, M., Hartmann, M., Branco, S., Wang, Z., Godhe, A., De Wit, P., Sánchez-García, M., Ebersberger, I., de Sousa, F., Amend, A.S., Jumpponen, A., Unterseher, M., Kristiansson, E., Abarenkov, K., Bertrand, Y.J.K., Sanli, K., Eriksson, K.M., Vik, U., Veldre, V. \& Nilsson, R.H. (2013) Improved software detection and extraction of ITS1 and ITS2 from ribosomal ITS sequences of fungi and other eukaryotes for analysis of environmental sequencing data ed M. Bunce. Methods in Ecology and Evolution.

Borges, Á.V., Saraiva, R.M. \& Maffia, L.A. (2015) Biocontrol of gray mold in tomato plants by Clonostachys rosea. Tropical Plant Pathology, 40, 71-76.

Breen, J.P. (1994) Acremonium endophyte interactions with enhanced plant resistance to insects. Annual review of entomology, 39, 401-423.

Bressan, W. \& Borges, M.T. (2004) Delivery methods for introducing endophytic bacteria into maize. BioControl, 49, 315-322.

Busby, P.E., Peay, K.G. \& Newcombe, G. (2016) Common foliar fungi of Populus trichocarpa modify Melampsora rust disease severity. New Phytologist, 209, 1681-1692.

Busby, P.E., Ridout, M. \& Newcombe, G. (2015) Fungal endophytes: modifiers of plant disease. Plant Molecular Biology, 90, 645-655. 
346 Buxdorf, K., Rahat, I. \& Levy, M. (2013) Pseudozyma aphidis induces ethylene-independent resistance in plants. Plant Signaling \& Behavior, 8, e26273.

348 Cáceres, M.D. \& Legendre, P. (2009) Associations between species and groups of sites: indices and statistical inference. Ecology, 90, 3566-3574.

350

351

352

353

354

355

356

357

358

359

360

361

362

363

364

365

366

367

368

369

370

371

372

373

374

375

376

377

Caporaso, J.G., Kuczynski, J., Stombaugh, J., Bittinger, K., Bushman, F.D., Costello, E.K., Fierer, N., Peña, A.G., Goodrich, J.K., Gordon, J.I., Huttley, G.A., Kelley, S.T., Knights, D., Koenig, J.E., Ley, R.E., Lozupone, C.A., McDonald, D., Muegge, B.D., Pirrung, M., Reeder, J., Sevinsky, J.R., Turnbaugh, P.J., Walters, W.A., Widmann, J., Yatsunenko, T., Zaneveld, J. \& Knight, R. (2010) QIIME allows analysis of high-throughput community sequencing data. Nature methods, 7, 335-336.

Caporaso, J.G., Lauber, C.L., Walters, W.A., Berg-Lyons, D., Lozupone, C.A., Turnbaugh, P.J., Fierer, N. \& Knight, R. (2011) Global patterns of 16S rRNA diversity at a depth of millions of sequences per sample. Proceedings of the National Academy of Sciences, 108, 4516-4522.

Desprez-Loustau, M.-L., Marçais, B., Nageleisen, L.-M., Piou, D. \& Vannini, A. (2006) Interactive effects of drought and pathogens in forest trees. Annals of Forest Science, 63, 597-612.

Edgar, R.C. (2010) Search and clustering orders of magnitude faster than BLAST. Bioinformatics, 26, 2460-2461.

Ferrazzano, S. \& Williamson, P.S. (2013) Benefits of mycorrhizal inoculation in reintroduction of endangered plant species under drought conditions. Journal of Arid Environments, 98, $123-125$.

Filonow, A.B., Vishniac, H.S., Anderson, J.A. \& Janisiewicz, W.J. (1996) Biological Control of Botrytis cinereain Apple by Yeasts from Various Habitats and Their Putative Mechanisms of Antagonism. Biological control, 7, 212-220.

Fu, S.-F., Sun, P.-F., Lu, H.-Y., Wei, J.-Y., Xiao, H.-S., Fang, W.-T., Cheng, B.-Y. \& Chou, J.Y. (2016) Plant growth-promoting traits of yeasts isolated from the phyllosphere and rhizosphere of Drosera spatulata Lab. Fungal Biology, 120, 433-448.

Gafni, A., Calderon, C.E., Harris, R., Buxdorf, K., Dafa-Berger, A., Zeilinger-Reichert, E. \& Levy, M. (2015) Biological control of the cucurbit powdery mildew pathogen Podosphaera xanthii by means of the epiphytic fungus Pseudozyma aphidis and parasitism as a mode of action. Frontiers in Plant Science, 6, 132. 
378 Gardes M., Bruns TD. 1993. ITS primers with enhanced specificity for basidiomycetesapplication to the identification of mycorrhizae and rusts. Molecular ecology 2:113-118.

380

381

382

383

384

385

386

387

388

389

390

391

392

393

394

395

396

397

398

399

400

401

402

403

404

405

406

407

Gemma, J.N., Koske, R.E. \& Habte, M. (2002) Mycorrhizal dependency of some endemic and endangered Hawaiian plant species. American Journal of Botany, 89, 337-345.

Glawe DA. 2007. First Report of Powdery Mildew of Stachys byzantina (Lamb's Ear) Caused by Neoerysiphe galeopsidis in North America. Plant Health Progress. DOI: 10.1094/PHP2007-1217-01-BR.

Hardoim, P. R., Overbeek, L. S. van, Berg, G., Pirttilä, A. M., Compant, S., Campisano, A., Sessitsch, A. (2015) The Hidden World within Plants: Ecological and Evolutionary Considerations for Defining Functioning of Microbial Endophytes. Microbiology and Molecular Biology Reviews, 79, 293-320

Harris, J. (2009) Soil Microbial Communities and Restoration Ecology: Facilitators or Followers? Science, 325, 573-574.

Hartley, S.E. \& Gange, A.C. (2009) Impacts of Plant Symbiotic Fungi on Insect Herbivores: Mutualism in a Multitrophic Context. Annual Review of Entomology, 54, 323-342.

Heilmann-Clausen, J., Barron, E.S., Boddy, L., Dahlberg, A., Griffith, G.W., Nordén, J., Ovaskainen, O., Perini, C., Senn-Irlet, B. \& Halme, P. (2015) A fungal perspective on conservation biology. Conservation Biology, 29, 61-68.

Heluta V., Takamatsu S., Harada M., Voytyuk S. 2010. Molecular phylogeny and taxonomy of Eurasian Neoerysiphe species infecting Asteraceae and Geranium. Persoonia : Molecular Phylogeny and Evolution of Fungi 24:81-92. DOI: 10.3767/003158510X501696.

Herre, E.A., Mejía, L.C., Kyllo, D.A., Rojas, E., Maynard, Z., Butler, A. \& Van Bael, S.A. (2007) ECOLOGICAL IMPLICATIONS OF ANTI-PATHOGEN EFFECTS OF TROPICAL FUNGAL ENDOPHYTES AND MYCORRHIZAE. Ecology, 88, 550-558.

The IUCN Red List of Threatened Species. Version 2017-2. Accessed: October 10, 2017

Karlsson, I., Friberg, H., Steinberg, C. \& Persson, P. (2014) Fungicide Effects on Fungal Community Composition in the Wheat Phyllosphere. PLOS ONE, 9, e111786.

Kiss, L. (2003) A review of fungal antagonists of powdery mildews and their potential as biocontrol agents. Pest management science, 59, 475-483. 
408

409

410

411

412

413

414

415

416

417

418

419

420

421

422

423

424

425

426

427

428

429

430

431

432 433

434

435

436

437

438

439

Kõljalg U., Nilsson RH., Abarenkov K., Tedersoo L., Taylor AFS., Bahram M., Bates ST., Bruns TD., Bengtsson-Palme J., Callaghan TM., Douglas B., Drenkhan T., Eberhardt U., Dueñas M., Grebenc T., Griffith GW., Hartmann M., Kirk PM., Kohout P., Larsson E., Lindahl BD., Lücking R., Martín MP., Matheny PB., Nguyen NH., Niskanen T., Oja J., Peay KG., Peintner U., Peterson M., Põldmaa K., Saag L., Saar I., Schüßler A., Scott JA., Senés C., Smith ME., Suija A., Taylor DL., Telleria MT., Weiss M., Larsson K-H. 2013. Towards a unified paradigm for sequence-based identification of fungi. Molecular Ecology 22, 5271-5277. DOI: 10.1111/mec.12481.

Lorenz, S., Guenther, M., Grumaz, C., Rupp, S., Zibek, S. \& Sohn, K. (2014) Genome sequence of the basidiomycetous fungus Pseudozyma aphidis DSM70725, an efficient producer of biosurfactant mannosylerythritol lipids. Genome announcements, 2, e00053-14.

Miller, T.C., Gubler, W.D., Laemmlen, F.F., Geng, S. \& Rizzo, D.M. (2004) Potential for using Lecanicillium lecanii for suppression of strawberry powdery mildew. Biocontrol Science and Technology, 14, 215-220.

Nilsson, R.H., Tedersoo, L., Ryberg, M., Kristiansson, E., Hartmann, M., Unterseher, M., Porter, T.M., Bengtsson-Palme, J., Walker, D.M., de Sousa, F., Gamper, H.A., Larsson, E., Larsson, K.-H., Kõljalg, U., Edgar, R.C. \& Abarenkov, K. (2015) A Comprehensive, Automatically Updated Fungal ITS Sequence Dataset for Reference-Based Chimera Control in Environmental Sequencing Efforts. Microbes and environments, 30, 145-150.

Okansen, J., Blanchet, F.G., Friendly, M., Kindt, R., Legendre, P., McGlinn, D., Minchin, P.R., O’Hara, R.B., Simpson, G.L., Solymos, P., Stevens, M.H.H., Szoecs, E. \& Wagner, H. (2016) Vegan: Community Ecology Package.

Petrini, O. (1986) Taxonomy of endophytic fungi of aerial plant tissues. Microbiology of the phyllosphere/edited by NJ Fokkema and J. van den Heuvel.

Porras-Alfaro, A. \& Bayman, P. (2011) Hidden Fungi, Emergent Properties: Endophytes and Microbiomes. Annual Review of Phytopathology, 49, 291-315.

Posada, F., Aime, M.C., Peterson, S.W., Rehner, S.A. \& Vega, F.E. (2007) Inoculation of coffee plants with the fungal entomopathogen Beauveria bassiana (Ascomycota: Hypocreales). Mycological Research, 111, 748-757.

Requena, N., Perez-Solis, E., Azcón-Aguilar, C., Jeffries, P. \& Barea, J.-M. (2001) Management of Indigenous Plant-Microbe Symbioses Aids Restoration of Desertified Ecosystems. Applied and Environmental Microbiology, 67, 495-498. 
440 Ridout, M. \& Newcombe, G. (2016) Disease suppression in winter wheat from novel symbiosis with forest fungi. Fungal Ecology, 20, 40-48.

442

443

444

445

446

447

448

449

450

451

452

453

454

455

456

457

458

459

460

461

462

463

464

465

466

467

468

469

Rigg, J.L., Offord, C.A., Zimmer, H., Anderson, I.C., Singh, B.K. \& Powell, J.R. (2016) Conservation by translocation: establishment of Wollemi pine and associated microbial communities in novel environments. Plant and Soil, 1-17.

Rodriguez, R.J., White Jr, J.F., Arnold, A.E. \& Redman, R.S. (2009) Fungal endophytes: diversity and functional roles. New Phytologist, 182, 314-330.

Rognes, T., Flouri, T., Nichols, B., Quince, C. \& Mahé, F. (2016) VSEARCH: a versatile open source tool for metagenomics. PeerJ, 4, e2584.

Rudgers, J.A., Fischer, S. \& Clay, K. (2010) Managing plant symbiosis: fungal endophyte genotype alters plant community composition. Journal of Applied Ecology, 47, 468-477.

Vicari, M., Hatcher, P.E. \& Ayres, P.G. (2002) Combined effect of foliar and mycorrhizal endophytes on an insect herbivore. Ecology, 83, 2452-2464.

Viterbo, A., Ramot, O., Chernin, L. \& Chet, I. (2002) Significance of lytic enzymes from Trichoderma spp. in the biocontrol of fungal plant pathogens. Antonie van Leeuwenhoek, 81, 549-556.

Weisenberger, L. \& Keir, M.J. (2012) A STATEWIDE ASSESSMENT OF EX SITU STATUS, CAPACITY AND NEEDS FOR THE CONSERVATION OF HAWAIIAN PLANTS. U.S. Fish and Wildlife Service.

White, T.J., Bruns, T., Lee, S., Taylor, J.W. \& others. (1990) Amplification and direct sequencing of fungal ribosomal RNA genes for phylogenetics. PCR protocols: a guide to methods and applications, 18, 315-322.

Xia, C., Zhang, X., Christensen, M.J., Nan, Z. \& Li, C. (2015) Epichloë endophyte affects the ability of powdery mildew (Blumeria graminis) to colonise drunken horse grass (Achnatherum inebrians). Fungal Ecology, 16, 26-33.

Zhang, J., Kobert, K., Flouri, T. \& Stamatakis, A. (2014) PEAR: a fast and accurate Illumina Paired-End reAd mergeR. Bioinformatics, 30, 614-620.

Zubek, S., Turnau, K., Tsimilli-Michael, M. \& Strasser, R.J. (2008) Response of endangered plant species to inoculation with arbuscular mycorrhizal fungi and soil bacteria. Mycorrhiza, 19, 113-123. 
Figure 1

Plant disease progression for both experimental rounds

Disease severity over time for each plant species during each experimental trial, measured as percentage of leaf area visibly infected by powdery mildew. Panels A and B - P. kaalaensis; Panels $C$ and D - P. mollis. The first trial (panels $A$ and $C$ ) lasted 30 days and the second trial (panels $B$ and D) lasted 90 days. P. kaalaensis plants receiving the whole leaf slurry had delayed infections and reduced overall infection severity (black lines). Error bars represent 95\% C.I. around the mean.

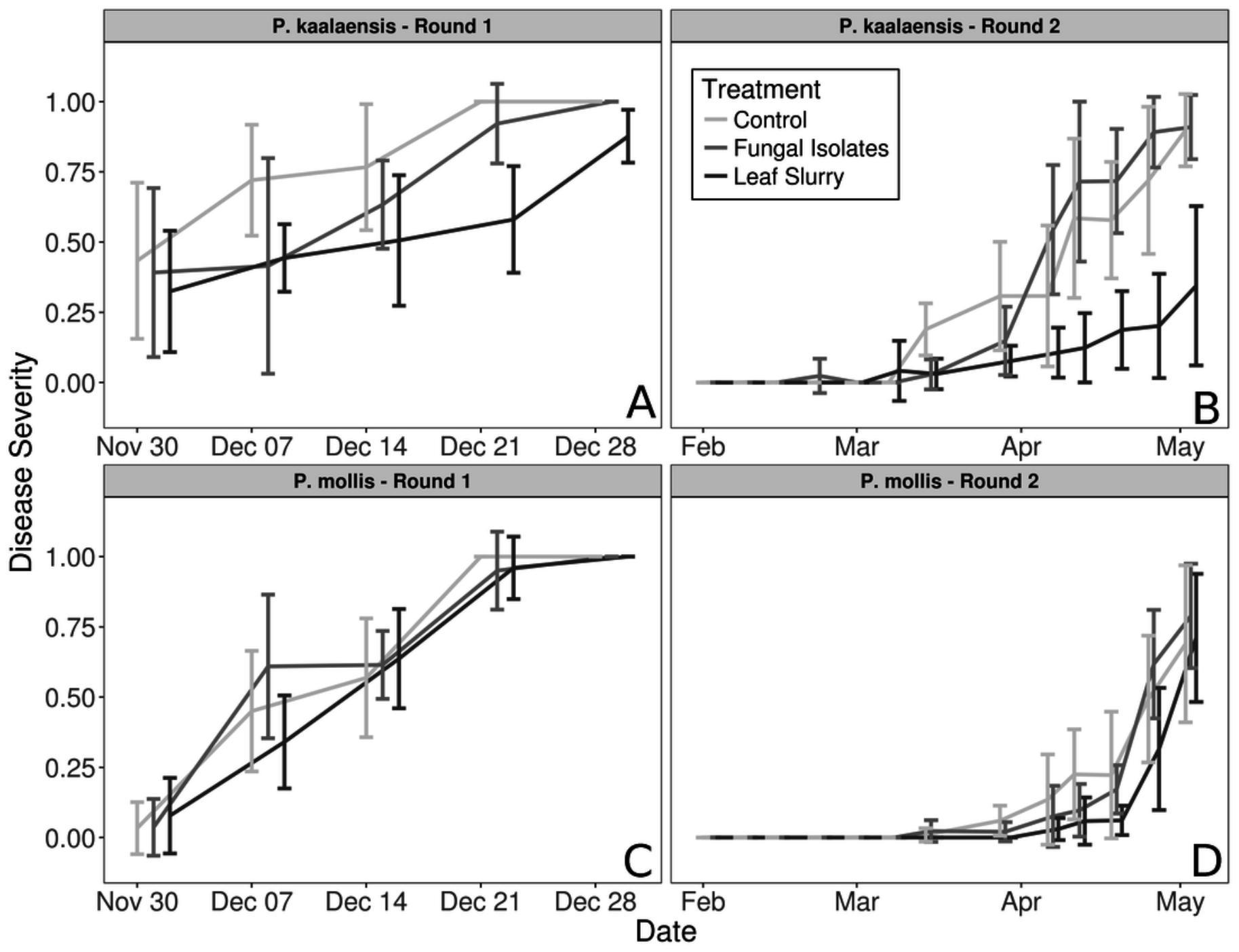


Figure 2

Taxonomic compositions of the two experimental donor inoculae

Species compositions (relative abundance) for each inoculum treatment, during both experimental trials. Both the fungal isolates (panel A) and the whole leaf slurries (panel B) were dominated by a single taxon. Taxonomy reflects assignments to the UNITE fungal ITS database.

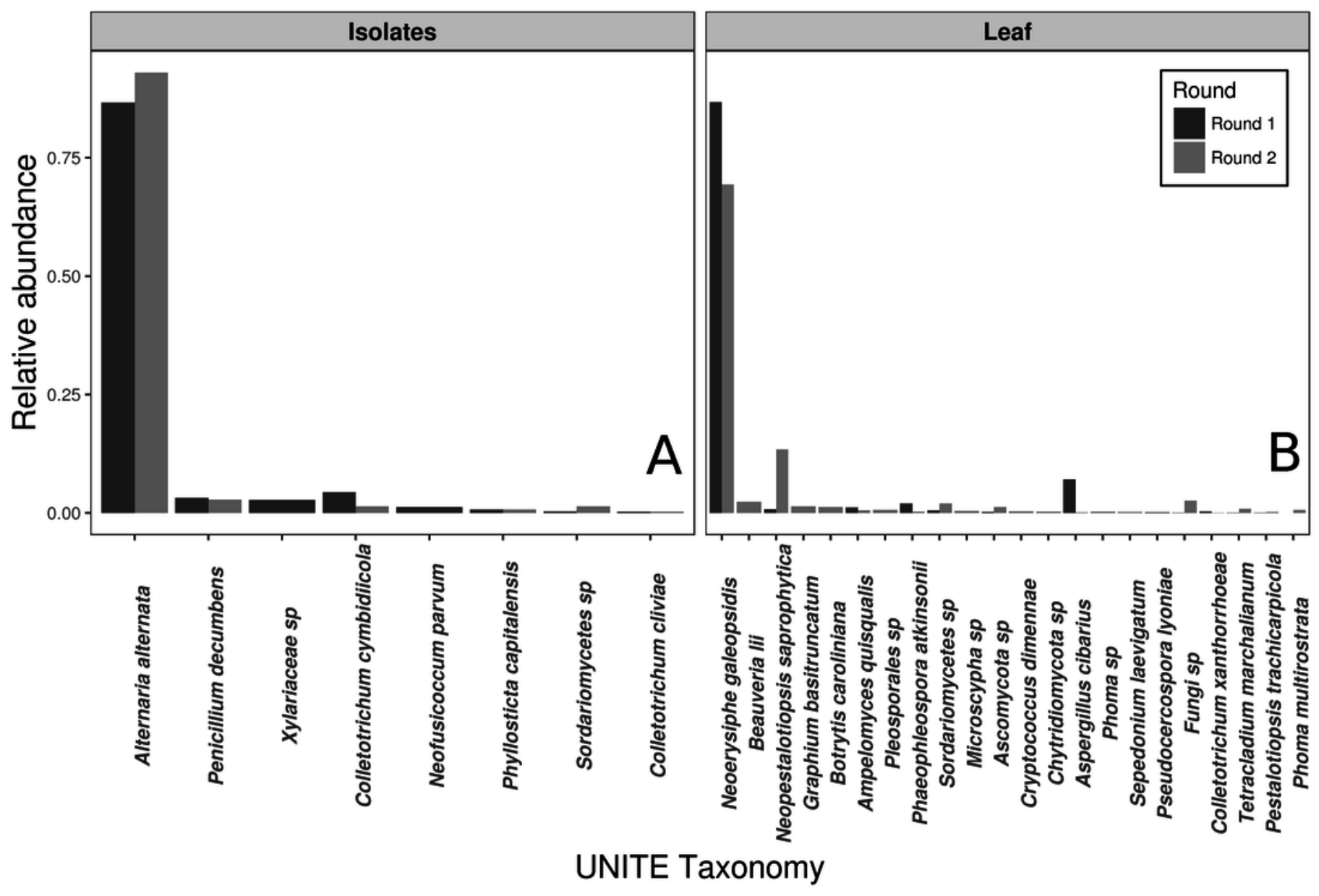




\section{Figure 3}

Disease severity as a function of $P$. aphidis abundance

N. galeopsidis infection severity as a function of $P$. aphidis abundance. Higher $P$. aphidis abundance was negatively correlated with infection severity. This figure shows data from $P$. kaalaensis observations over both experimental rounds. Line represents loess smoothing and gray area represents $95 \%$ C.I. around the mean.

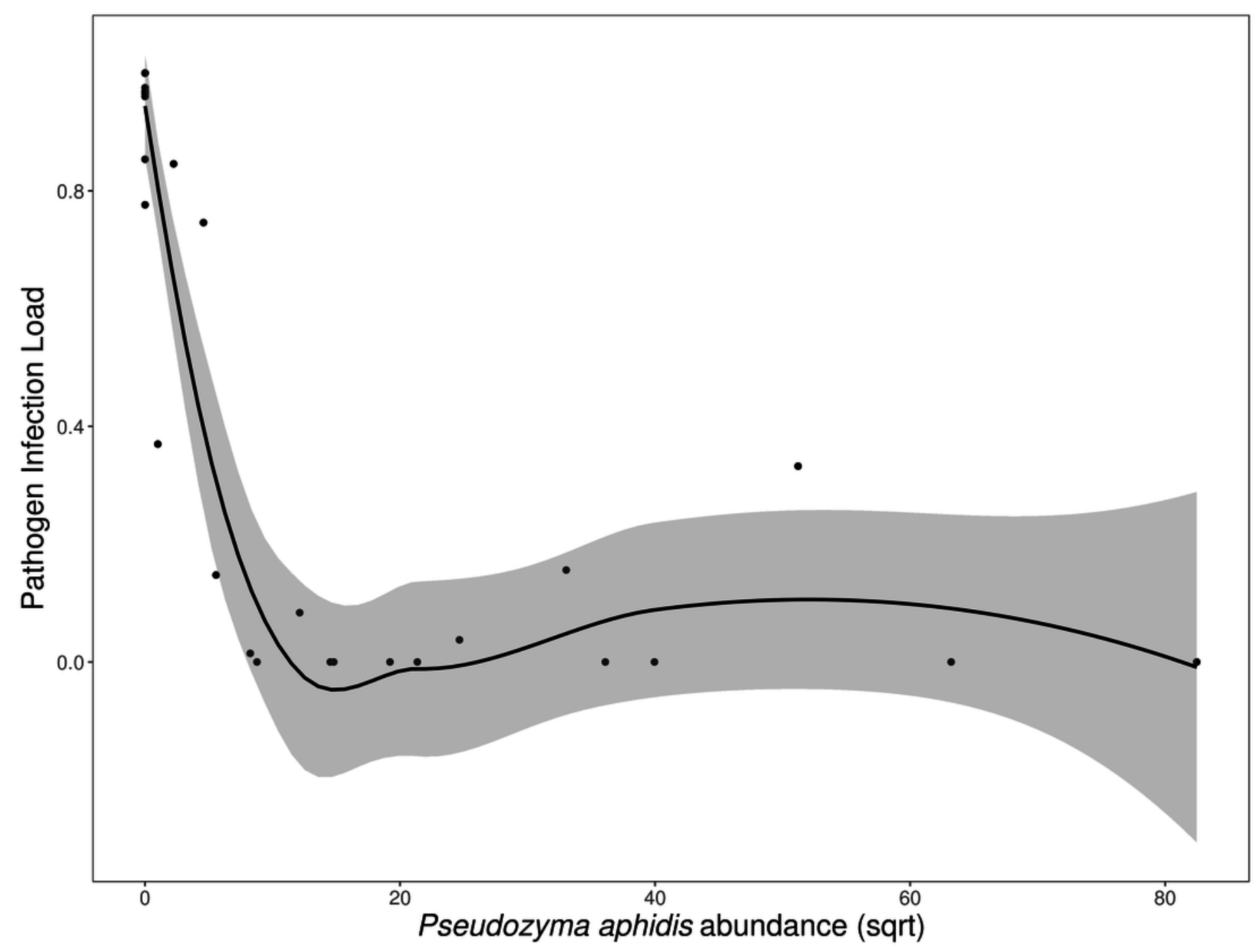

